\title{
Transient localization in crystalline organic semiconductors
}

\author{
S. Ciuchi, ${ }^{1}$ S. Fratini, ${ }^{2}$ and D. Mayou ${ }^{2}$ \\ ${ }^{1}$ Istituto dei Sistemi Complessi CNR, CNISM and Dipartimento di Fisica, Università dell'Aquila, via Vetoio, I-67100 Coppito-L'Aquila, Italy \\ ${ }^{2}$ Institut Néel-CNRS and Université Joseph Fourier, Boîte Postale 166, F-38042 Grenoble Cedex 9, France
}

(Received 20 January 2011; published 23 February 2011)

\begin{abstract}
A relation derived from the Kubo formula shows that optical conductivity measurements below the gap frequency in doped semiconductors can be used to probe directly the time-dependent quantum dynamics of charge carriers. This allows to extract fundamental quantities such as the elastic and inelastic scattering rates, as well as the localization length in disordered systems. When applied to crystalline organic semiconductors, an incipient electron localization caused by large dynamical lattice disorder is unveiled, implying a breakdown of semiclassical transport.
\end{abstract}

DOI: 10.1103/PhysRevB.83.081202

PACS number(s): 72.40.+w, 71.38.-k, 72.20.Dp, 72.80.Le

Introduction. "Bad" conductors are systems presenting a breakdown of the semiclassical Bloch-Boltzmann description of electronic transport. Known examples are found in various classes of materials such as disordered systems, ${ }^{1}$ transition metal compounds, ${ }^{2}$ alkali-doped and charge-transfer organic metals, ${ }^{3}$ and quasicrystals. ${ }^{4,5}$ In all these cases, the electron motion is so much slowed down (by disorder, electronic correlations, polaronic effects, or by structural constraints) that the semiclassical assumption of welldefined wave packets undergoing rare scattering events is not valid.

An analogous situation is encountered in crystalline organic semiconductors. There, a fundamental unsettled question is whether the mechanism of charge transport can be ultimately understood from the point of view of band electrons alone, as suggested by the "band-like" temperature dependence of the measured mobility. In these materials, the inherently large thermal molecular motions act as strong electron scatterers, leading to apparent electron mean-free paths comparable or even smaller than the intermolecular distances. While a generalization of semiclassical transport theory has been recently proposed in Ref. 6 to deal with this situation, it might well be that the very nature of semiclassical transport is insufficient to appropriately describe the charge transport mechanism in these materials. Indeed, recent numerical studies have suggested a radically different point of view based on a form of electron localization due to the dynamical disorder caused by the thermal molecular motion. ${ }^{7,8}$ Accordingly, a theory of electron transport in organic semiconductors would require a proper account of quantum corrections to the electron dynamics, not included in semiclassical treatments.

In this Rapid Communication we tackle this problem by expressing the Kubo formula as a relation between the optical conductivity and the time-resolved quantum dynamics of electrons. We first consider a microscopic model with dynamical lattice disorder that illustrates the characteristic behavior of the quantum diffusion. A relaxation time approximation is then introduced that treats the effect of inelastic scattering by low-frequency lattice vibrations in an intuitive way. Finally these concepts are used in the interpretation of experimental data in crystalline rubrene, providing evidence for localization effects.
Formalism. The quantum diffusion of electrons in a given spatial direction can be measured via their quantummechanical spread

$$
\Delta X^{2}(t)=\left\langle[\hat{X}(t)-\hat{X}(0)]^{2}\right\rangle,
$$

where $\hat{X}(t)=\sum_{i=1}^{N} \hat{x}_{i}(t)$ is the total position operator of $N$ electrons in the Heisenberg representation and $\langle\cdots\rangle=$ $\operatorname{Tr}\left[e^{-\beta H}(\ldots)\right] / Z$ denotes the thermodynamic average. $\Delta X^{2}(t)$ is directly related to the symmetrized self-correlation function of the velocity operator $\hat{V}_{X}(t)=\frac{d \hat{X}(t)}{d t}, C(t)=\left\langle\hat{V}_{X}(t) \hat{V}_{X}(0)+\right.$ $\left.\hat{V}_{X}(0) \hat{V}_{X}(t)\right\rangle, \operatorname{via}^{4,5}$

$$
\frac{d \Delta X^{2}(t)}{d t}=\int_{0}^{t} C\left(t^{\prime}\right) d t^{\prime}
$$

On the other hand, the Kubo formula expresses the dissipative part of the optical conductivity at $\omega \neq 0$ as

$$
\sigma(\omega)=\frac{e^{2}}{v \hbar \omega} \operatorname{Re} \int_{0}^{\infty} e^{i \omega t}\left\langle\left[\hat{V}_{X}(t), \hat{V}_{X}(0)\right]\right\rangle d t,
$$

where $v$ is the volume of the system. This can be exactly related to the symmetrized $C(t)$ of Eq. (2) and therefore to the quantum diffusion $\Delta X^{2}(t)$. Replacing the commutator in Eq. (3) with the anticommutator of Eq. (2) can be absorbed into a detailed-balance prefactor, yielding

$$
\sigma(\omega)=-\frac{e^{2} \omega^{2}}{v} \frac{\tanh (\beta \hbar \omega / 2)}{\hbar \omega} \operatorname{Re} \int_{0}^{\infty} e^{i \omega t} \Delta X^{2}(t) d t,
$$

with $\beta=1 / k_{B} T$ (see Refs. 9 and 10 for related aspects). The above Eq. (4) is a restatement of the Kubo formula, identifying the time-dependent quantum diffusion as the physical quantity that is dual to the optical absorption in the frequency domain. For independent nondegenerate electrons, the formalism presented above acquires an intuitive meaning in terms of the quantum spread of the electronic wave functions, as in this case one has $\Delta X^{2}(t)=N \Delta x^{2}(t)$, with $\Delta x^{2}(t)$ referring to each individual particle. Equation (4) can be inverted to give

$$
\Delta x^{2}(t)=\frac{2 \hbar}{\pi e^{2}} \operatorname{Re} \int_{0}^{\infty}\left(1-e^{-i \omega t}\right) \frac{\sigma(\omega) / n}{\omega \tanh (\beta \hbar \omega / 2)} d \omega,
$$

with $n=N / v$ the electron density. 
Microscopic description of lattice disorder. To set the concepts clearly we apply the quantum diffusion formalism to the following Hamiltonian ${ }^{6,7}$

$$
H=-J \sum_{i}\left[1-\alpha\left(u_{i}-u_{i+1}\right)\right]\left(c_{i}^{+} c_{i+1}+c_{i+1}^{+} c_{i}\right)+H_{p h} .
$$

In this model, electrons in a one-dimensional tight-binding band have their intermolecular transfer integrals modulated by molecular vibrations of frequency $\omega_{0}$, represented by $H_{p h}=\sum_{i} \frac{M \omega_{0}^{2} u_{i}^{2}}{2}+\frac{p_{i}^{2}}{2 M}$. Equation (6) captures the effects of strong dynamical lattice disorder that seem to be crucial in crystalline organic semiconductors. The phonon frequency $\omega_{0}$ is small due to the large molecular weight, so that the lattice fluctuations can be treated classically $\left(k_{B} T>\hbar \omega_{0}\right)$. Their coupling to electrons is governed by the dimensionless parameter $\lambda=\alpha^{2} J /\left(2 M \omega_{0}^{2}\right)$.

To calculate the electron diffusion $\Delta x^{2}(t)$ in the presence of lattice dynamics we employ mixed quantum-classical simulations based on the Ehrenfest coupled equations ${ }^{7}$ on a 1024-site chain. We solve the Schrödinger equation and average over up to 12800 initial conditions, with the initial displacements $u_{i}$ obeying a thermal distribution $P\left(u_{i}\right) \propto$ $\exp \left(-M \omega_{0}^{2} u_{i}^{2} / 2 k_{B} T\right)$. The case of a frozen disordered lattice is treated by averaging over the same set of disorder realizations (see Ref. 6). In the latter case, the results are cross checked via an exact diagonalization of the electronic problem on 256 sites, allowing for a direct verification of Eqs. (4) and (5).

Figure 1 shows the electron spread $\Delta x(t) \equiv \sqrt{\Delta x^{2}(t)}$ and the time-dependent diffusivity $D(t)=\frac{d \Delta x^{2}(t)}{2 d t}$ for a representative choice of microscopic parameters: $\lambda \stackrel{2 d t}{=} 0.25, J=0.11 \mathrm{eV}$, $T=0.235 \mathrm{~J}=300 \mathrm{~K}$, and different values of $\omega_{0}$. The dashed line in Fig. 1(a) is the result for static disorder $\omega_{0}=0$, showing a finite localization length $L=3.6 a$. The corresponding $D(t)$ in Fig. 1(b) increases at short times in the ballistic regime, then exhibits oscillations. At subsequent times the oscillations are damped and $D(t)$ steadily decreases and vanishes. Following Eq. (2), $2(d D / d t)=C(t)$ is precisely the velocity correlation function: a negative slope is therefore signaling the occurrence of backscattering underlying the phenomenon of Anderson localization [in a classical picture, $C(t)<0$ implies that the velocity at time $t$ is opposite to its value at time $t=0$ ]. This occurs, as expected, at times greater than the elastic scattering time, ${ }^{1}$ which is given by $\tau_{\mathrm{el}}=(\pi \lambda T)^{-1}=5.4$ in the present units. ${ }^{6}$

The results in the presence of lattice dynamics $\left(\omega_{0} \neq 0\right)$ closely follow the localized behavior at short and intermediate times. However, upon reaching the time scale of lattice vibrations, $1 / \omega_{0}$ (indicated by arrows), localization is destroyed and $\Delta x(t)$ starts increasing indefinitely. The existence of a transient localization phenomenon at times $\tau_{\mathrm{el}} \lesssim t \lesssim 1 / \omega_{0}$ is one of the main results of this work. It indicates that the electronic transport mechanism is markedly nonsemiclassical, the final outcome being determined by the characteristic time scale of lattice disorder.

As a side remark, Fig. 1(b) illustrates a fundamental drawback of the Ehrenfest method, which makes it inadequate to determine asymptotically the electron diffusion: The diffusivity does not apparently tend to a constant value but rather exhibits
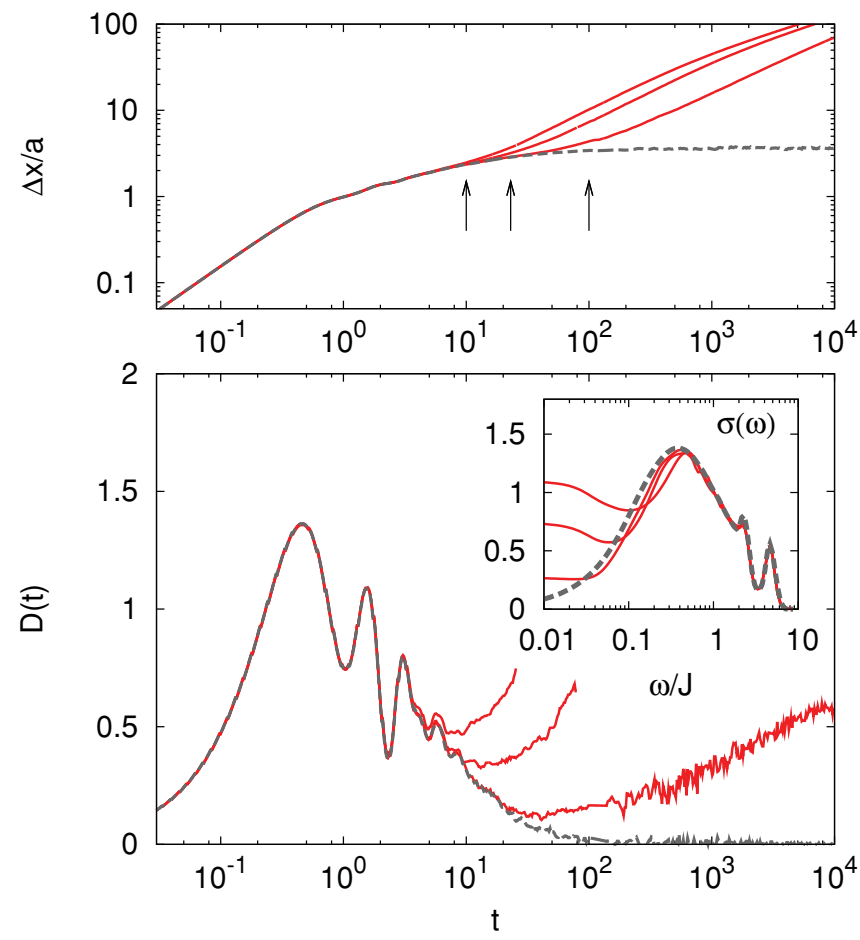

FIG. 1. (Color online) (a) Quantum spread obtained from the microscopic model Eq. (6) for static (gray, dashed) as well as dynamical disorder (red, full lines: from bottom to top, $\hbar \omega_{0} / J=$ $0.01,0.0435,0.1)$. Times are in units of $\hbar / J$. (b) The corresponding instantaneous diffusivity $D(t)=\left(d \Delta x^{2} / d t\right) / 2$. The inset shows the optical conductivity obtained via Eq. (4).

an upward drift at long times. The total energy of the system (not shown) is conserved in the simulation with a relative precision of $2 \cdot 10^{-7}$, which rules out possible integration errors. The origin of this spurious phenomenon rather lies in the fact that the Ehrenfest equations do not properly conserve the Maxwell-Boltzmann statistical distribution: ${ }^{11}$ The repeated action of the lattice vibrations (an external, time-dependent potential) eventually drives the electrons to an arbitrarily high effective temperature, a fact that could be at the origin of the $T^{-2}$ temperature dependence of the mobility obtained by this method. . $^{72,13}$

The inset of Fig. 1(b) shows the optical conductivity $\sigma(\omega)$ calculated by applying Eq. (4) to the data of Fig. 1(a), neglecting the spurious superdiffusive behavior at long times. The result of the static disorder problem $\left(\omega_{0}=0\right)$ is shown for reference (gray, dashed). We see that the dynamical nature of the lattice only modifies the low frequency region of the spectrum $\omega \lesssim \omega_{0}$. It does not affect substantially the localization peak at $\omega_{\text {loc }} \simeq 0.4 \mathrm{~J}$ as long as $\omega_{0} \ll \omega_{\text {loc }}$, nor the absorption band at higher frequencies.

Relaxation time approximation. To understand how localization features can actually coexist with a diffusive behavior at long times, we now implement the relaxation time approximation (RTA) as a simple scheme bridging between localization and diffusion. The idea underlying the RTA is to express the dynamical properties of the actual system in terms of those of a suitably defined reference system, from which 
it decays over time. Specifically, defining $C_{0}$ as a reference velocity correlation function, the relation

$$
C_{\mathrm{RTA}}(t)=C_{0}(t) e^{-t / \tau},
$$

describes the damping of velocity correlations caused by relaxation processes with a characteristic time $\tau .^{4,5,14}$ In the semiclassical theory of electron transport, one starts from a perfectly periodic crystal and describes via Eq. (7) the momentum relaxation due to the scattering of Bloch states. In that case $C_{0}=2 v_{\mathrm{avg}}^{2}$ is a constant (twice the thermal average of the squared band velocity) and the resulting diffusivity, $D_{\mathrm{RTA}}(t)=v_{\mathrm{avg}}^{2} \tau\left[1-e^{-t / \tau}\right]$, is a monotonically increasing function of time.

One can alternatively take a localized system with static disorder as the reference state, as suggested by Fig. 1. At times shorter than the typical time scale of the lattice motion, $\tau_{\text {in }} \sim$ $1 / \omega_{0}$, the molecular lattice appears to the moving electrons as an essentially frozen, disordered landscape. In this case, nothing prevents the buildup of quantum interferences that lie at the origin of Anderson localization. The velocity correlation function $C(t)$ then initially coincides with that of a system with static disorder $C_{0}(t)$. However, quantum interferences are destroyed at longer times because, due to the lattice dynamics, the electrons encounter different disorder landscapes when moving in the forward and backward directions: ${ }^{1}$ Eq. (7) is the simplest form that is able to capture such a decay process. From Eqs. (2) and (4) it is easy to see that, starting from a localized system [i.e., one with a vanishing diffusion constant, $\left.\int_{0}^{\infty} C_{0}(t) d t=0\right]$, Eq. (7) restores a finite diffusion constant $D_{\text {RTA }}=L_{0}^{2}\left(\tau_{\text {in }}\right) /\left(2 \tau_{\text {in }}\right)$, which is analogous to the Thouless diffusivity of Anderson insulators. ${ }^{15}$ This value is essentially equal to the diffusivity of the reference localized system at a time $t \approx \tau_{\text {in }}$. The quantity $L_{0}^{2}\left(\tau_{\text {in }}\right)=\int e^{-t / \tau_{\text {in }}} \Delta x_{0}^{2}(t) d t / \tau_{\text {in }}$ represents the typical electron spread achieved at a time $\tau_{\text {in }}$, before diffusion sets back in. It therefore acquires the meaning of a transient localization length. The emerging physical picture is that of electrons prone to localization, but that can take advantage of the lattice motion to diffuse freely over a distance $L_{0}\left(\tau_{\text {in }}\right)$, with a trial rate $1 / \tau_{\text {in }}$.

From Eqs. (4) and (7) we obtain a mobility

$$
\mu(T) \simeq \frac{e}{k_{B} T} \frac{L_{0}^{2}\left(\tau_{\mathrm{in}}\right)}{2 \tau_{\mathrm{in}}} .
$$

Although a systematic study of the electron mobility of organic semiconductors is beyond the scope of this work, we note here that under quite general assumptions Eq. (8) implies a power-law temperature dependence $\mu \sim T^{-\alpha}$, even though the microscopic transport mechanism is far from a conventional band transport (the exponent $\alpha$ depends on how the transient localization length varies with the thermal lattice disorder).

Real time dynamics from experiment. We now show how optical conductivity experiments can provide direct information on the relevant time and length scales of the problem. Figure 2 reports the instantaneous diffusivity $D(t)$ in the direction of highest conduction of rubrene, obtained via Eq. (5) by direct integration of the data of Ref. 16 (analogous results are obtained from Ref. 17). $D(t)$ increases first, reaches a maximum, and then decreases by a factor of 3 before

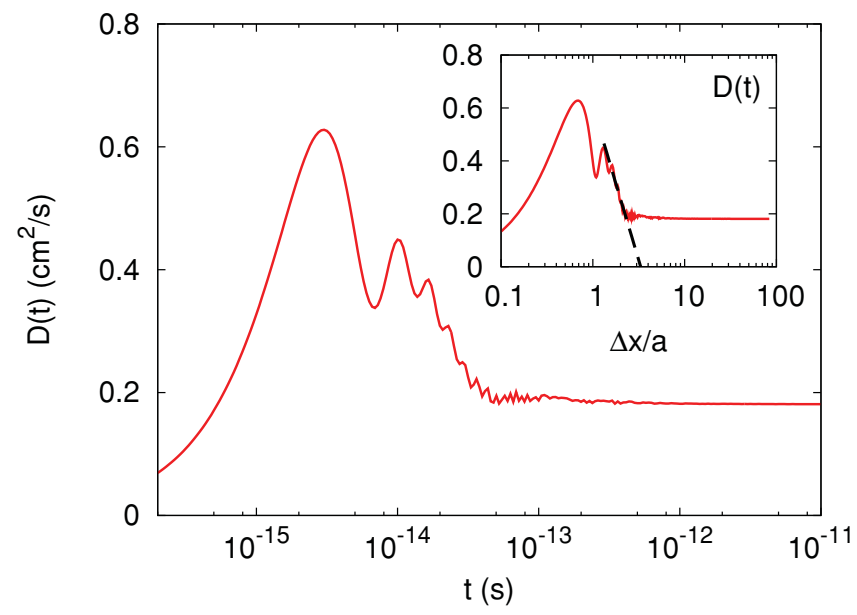

FIG. 2. (Color online) Time-dependent electron diffusivity $D(t)$ extracted via Eq. (4) from the experimental optical conductivity of Ref. 16 in the direction of highest conduction. The absolute value is fixed by the measured mobility $\mu \simeq 7 \mathrm{~cm}^{2} / V s$. The inset shows the same quantity as a function of the instantaneous electron spread. The dashed line is the weak localization extrapolation.

stabilizing to a constant value. The shape of the diffusivity curve is remarkably similar to the theoretical result of Fig. 1 and hardly compatible with the semiclassical picture discussed after Eq. (7) in which $D(t)=v_{\text {avg }}^{2} \tau\left[1-e^{-t / \tau}\right]$ increases and directly saturates. This suggests that, as in the model calculation, the decrease of $D(t)$ is due to localization effects occurring at times shorter than the lattice dynamics. An elastic scattering time of the order of $\tau_{\mathrm{el}} \approx 10^{-14} \mathrm{~s}$ can be tentatively identified with the region of negative slope in Fig. 2. According to the arguments given above, a diffusive regime $[D(t)=$ constant] sets up at time scales beyond the inelastic scattering time. This is what Fig. 2 reveals with $\tau_{\text {in }} \approx 5 \cdot 10^{-14} s$.

The inset shows a plot of $D(t)$ as a function of $\Delta x(t)$ and gives access to the relevant length scales. We find for

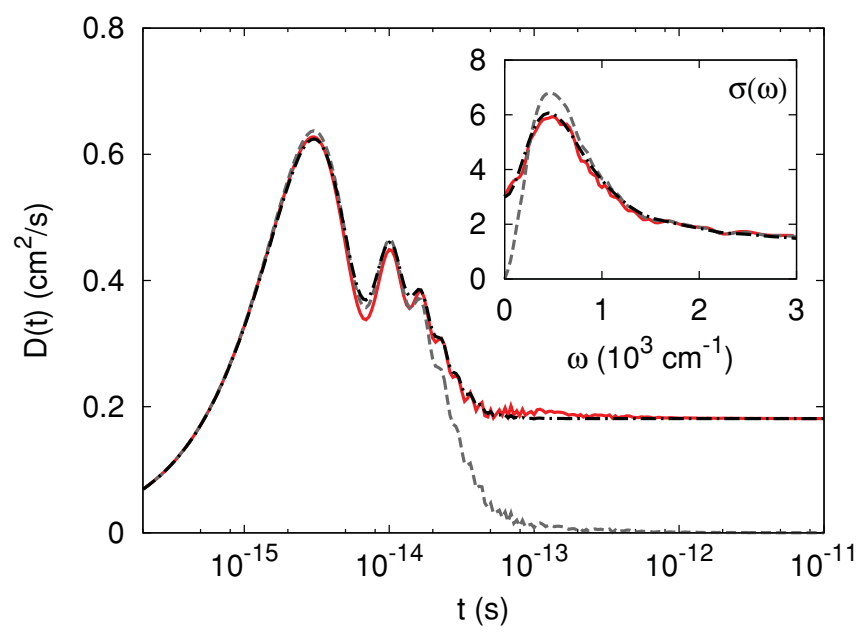

FIG. 3. (Color online) The experimental diffusivity of Fig. 2 (red, continuous line) is compared with the result of the RTA (black, dashdotted line) and the localization ansatz defined in the text (gray, dashed line). The inset shows the experimental optical conductivity of Ref. 16 together with the RTA and the localization ansatz (arb. units). 
the elastic and inelastic mean-free paths $\ell_{\mathrm{el}} \approx a$ and $\ell_{\mathrm{in}} \approx 3 a$, respectively, with $a=7.2 \AA$ the intermolecular distance. In addition, this inset suggests that without inelastic scattering (i.e., for a fixed set of disordered molecular positions) the diffusivity $D(t)$ would extrapolate to zero at a localization length of the order of 3-5 intermolecular distances. Note that the linear extrapolation of diffusivity with the $\log$ of the length is a standard approximation for twodimensional systems. ${ }^{1}$ Yet rubrene should be considered as intermediate between one- and two-dimensional due to its highly anisotropic character. ${ }^{18}$ For a one-dimensional system localization sets in more efficiently therefore the above extrapolation should be an upper bound to the true localization length.

We finally show in Fig. 3 how the RTA can be used to extract quantitative microscopic information from the optical data. One first constructs an ansatz for the reference conductivity $\sigma_{0}(\omega)$ representing the ideal case with frozen disorder (i.e., no inelastic scattering). This can be done starting from the experimental optical absorption (inset: red, full line), by enforcing the condition $\sigma_{0}(\omega \rightarrow 0)=0$ appropriate for a localized system (gray, dashed line). The RTA result (black, dash-dotted line) is then obtained by applying Eq. (7) to fit the experimental curve. The optical conductivity and the resulting diffusivity both nicely agree with the experimental data. The fitting procedure yields $\tau_{\text {in }}=5.1 \cdot 10^{-14} s$, corresponding to a frequency $\omega_{\text {in }}=104 \mathrm{~cm}^{-1}$, consistent with the relevant intermolecular phonon frequencies in rubrene. ${ }^{19,20}$ From the same fit, the estimated transient localization length in the direction of highest conduction is $L_{0}\left(\tau_{\text {in }}\right) \simeq 2 a$ [the localization length of the static ansatz is $L_{0}(t \rightarrow \infty) \simeq 3 a$ ] The present analysis shows that the finite frequency absorption peak observed in rubrene ${ }^{16,17}$ should be ascribed to transient localization effects (i.e., occurring before the dynamics of the lattice set in) and constitute a signature of an unconventional transport mechanism.

Concluding remarks. The relation between the quantum dynamics of electrons and the optical conductivity that stems from the Kubo formula appears to be a powerful tool to analyze the charge dynamics in semiconductors with unconventional transport properties. When applied to experimental data on crystalline organic semiconductors, it provides evidence for the role played by localization phenomena in the charge transport mechanism. The scenario emerging from the above analysis is indicative of a prominent role of the dynamical lattice disorder, which is supported by a microscopic calculation on a one-dimensional model.

Acknowledgment. S.C. acknowledges useful discussions with Sara Bonella.
${ }^{1}$ P. A. Lee and T. V. Ramakrishnan, Rev. Mod. Phys. 57, 287 (1985).

${ }^{2}$ O. Gunnarsson, M. Calandra, and J. E. Han, Rev. Mod. Phys. 75, 1085 (2003).

${ }^{3}$ K. Takenaka, M. Tamura, N. Tajima, H. Takagi, J. Nohara, and S. Sugai, Phys. Rev. Lett. 95, 227801 (2005).

${ }^{4}$ D. Mayou, Phys. Rev. Lett. 85, 1290 (2000).

${ }^{5}$ G. Trambly de Laissardière, J.-P. Julien, and D. Mayou, Phys. Rev. Lett. 97, 026601 (2006).

${ }^{6}$ S. Fratini and S. Ciuchi, Phys. Rev. Lett. 103, 266601 (2009).

${ }^{7}$ A. Troisi and G. Orlandi, Phys. Rev. Lett. 96, 086601 (2006).

${ }^{8}$ J.-D. Picon, M. N. Bussac, and L. Zuppiroli, Phys. Rev. B 75, 235106 (2007).

${ }^{9}$ N. H. Lindner and A. Auerbach, Phys. Rev. B 81, 054512 (2010).

${ }^{10}$ R. Kubo, M. Toda, and N. Hashitsume, Statistical Physics II (Springer, New York, 1991).

${ }^{11}$ P. V. Parandekar and J. C. Tully, J. Chem. Phys. 122, 094102 (2005).

${ }^{12}$ A. Troisi, Adv. Mat. 19, 2000 (2007).
${ }^{13}$ This indeed corresponds to the result obtained by the Boltzmann theory at unphysical temperatures higher than the bandwidth [L. Friedman, Phys. Rev. 133, A1668 (1964)].

${ }^{14}$ Equation (7) is equivalent to performing a convolution of $C_{0}(\omega)$ with a lorentzian of width $1 / \tau$, not a convolution of the conductivity $\sigma_{0}(\omega)$ as is often done in the literature.

${ }^{15}$ D. J. Thouless, Phys. Rev. Lett. 39, 1167 (1977).

${ }^{16}$ Z. Q. Li, V. Podzorov, N. Sai, M. C. Martin, M. E. Gershenson, M. DiVentra, and D. N. Basov, Phys. Rev. Lett. 99, 016403 (2007).

${ }^{17}$ M. Fischer, M. Dressel, B. Gompf, A. K. Tripathi, and J. Pflaum, Appl. Phys. Lett. 89, 182103 (2006).

${ }^{18}$ S. I. Machida, Y. Nakayama, S. Duhm, Q. Xin, A. Funakoshi, N. Ogawa, S. Kera, N. Ueno, and H. Ishii, Phys. Rev. Lett. 104, 156401 (2010).

${ }^{19}$ Z. Q. Ren, L. E. McNeil, S. Liu, and C. Kloc, Phys. Rev. B 80, 245211 (2009).

${ }^{20}$ A. Girlando, L. Grisanti, M. Masino, I. Bilotti, A. Brillante, R. G. DellaValle, and E. Venuti, Phys. Rev. B 82, 035208 (2010). 